http://dx.doi.org/10.21611/qirt.1994.022

\title{
Infrared thermography study of a thermal anti-icing system
}

\author{
BY BUCHLIN J-M. *, PRÉTREL H. *, PLANQUART H.*, LANGER H. and THIRY F.॰
}

* von Karman Institute for Fluid Dynamics, Rhode-Saint-Genèse, Belgium

- SONACA, Gosselies, Belgium

\section{Abstract}

This paper deals with the mapping of the convective heat transfer in a multijet anti-icing system by application of the quantitative infrared thermography technique. The experiments are conducted on a full-scale mock-up of the leading edge section of a jet aircraft wing. A steady state infrared thermography technique combined with the heating foil method is developed and successfully applied to recover the complete distribution of the thermal exchange coefficient on the concave surface of the skin. The heating performance of such a multijet system depends on the jet Reynolds number, the stand-off distance of the supply duct and the spanwise and chordwise jet arrangement. Tridimensional numerical simulations are performed with the FLUENT code. Despite the complexity of the flow phenomena and the stiff character of the boundary conditions involved, satisfactory agreement is found between the predictions and the IR data.

\section{Introduction}

The ability of impinging jets to produce high local transport coefficients has initiated numerous industrial and engineering applications of this type of flow to situations where heat and/or mass transfer prevail [1]. Recent designs of anti-icing systems of wing and tail surfaces of airplanes are based on the aerothermal effect of impinging jets [2]. In such a technique, hot jets are issued from a perforated supply duct called a "piccolo"' as sketched in figure 1. The hot air flowing on the inner surfaces of the skin prevents the ice formation on the leading edge when adverse flight conditions are experienced.

The design of a thermal anti-icing unit requires identifying the key parameters that control the convective heat transfer coefficient of the multijet system. Such a problem can be examined via an experimental approach involving up-to-date thermo-optical nonintrusive techniques. Recent progress in the field of infrared imaging has initiated the development and application of IR thermography to fluid flow problems [3]. The suitability of such a technique to complex flow situations such as multijets has already been

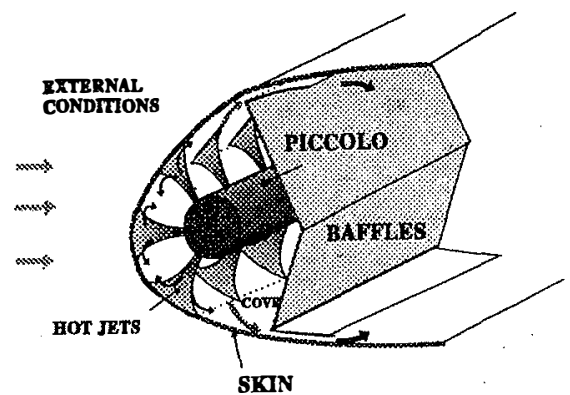

Fig.1-Principle of the anti-icing by hot jets demonstrated [4]. The present paper constitutes an additional contribution in illustrating the applicability of the quantitative IR thermography to outline the convective heat transfer distribution on a concave surface such as the one formed by the leading edge of a wing. After describing the experimental set-up and the procedure to collect the thermal data, the paper emphasizes the effect of the flow and geometrical parameters on the distribution. of the convective heat transfer coefficient. 
http://dx.doi.org/10.21611/qirt.1994.022

Finally, the experimental observations are compared to tridimensional numerical simulations to appraise the applicability of CFD codes to such a flow problem.

\section{Test apparatus and experimental procedure}

The experimental model consists of a leading edge section at scale one of a regional jet wing. A sketch of the mock-up is proposed in figure 2. It is made of a Plexiglas foil, $1 \mathrm{~mm}$ thick and $0.40 \mathrm{~m}$ long. Two baffles located on the rear of the model delimit the zone air flow called the cove. The two lateral ends of the model and the baffles are insulated by $5 \mathrm{~cm}$ of polystyrene to minimize the thermal loss. The supply piccolo duct is a titanium tube closed at its extremity and perforated by a set of sharp orifices. The jet arrangements tested are composed of three or four rows of holes. They are defined by the chordwise and spanwise jet spacing $S c$ and $S p$ respectively as indicated in figure 3 . The jets impinge on the internal surface of the airfoil and the gas flow exhausts through two thin slits pictured in figure 2.

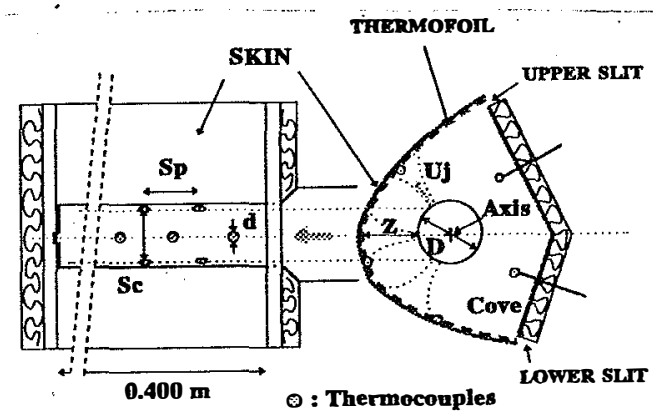

Fig. 2 - Sketch of the mock-up

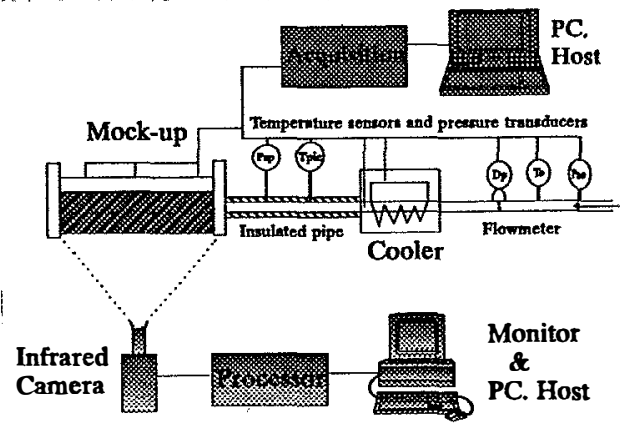

Fig. 3 - Sketch of the test set--up

The internal surface of the model is covered with a perfect thermal contact by a thin thermofoil, $0.5 \mathrm{~mm}$ thick, specially designed to provide constant and uniform heat flux. The jet is cooled to ensure a substantial temperature difference between the skin and the jet. This inverse situation compared to the real case, does not affect the mechanism of convective heat transfer which is based only on the magnitude of the temperature difference. The model is instrumented with a set of thermocouples to measure the temperature of air in the cove and at the slit exit and at some locations on the skin surface. The jet stand-off distance is varied by using different diameters of the piccolo always centered on the model axis.

Dry compressed air feeds the piccolo tube through a pipe implemented with a gas cooler device and instrumented with a flowmeter, pressure transducers and temperature sensors to monitor the flow rate and the air temperature. The probes are connected to a PC controlled data acquisition system. The technique to recover the internal temperature map is essentially similar to that used for the study reported in reference [4] concerning heat transfer of multijets on a flat plate: the external surface of the model is scanned by an Agema 900 infrared camera equipped with an $\mathrm{HgCdTe}$ detector sensitive in the 8-12 $\mathrm{\mu m}$ wavelength range and cooled by liquid nitrogen. The standard optical set-up is $10^{\circ}$ vertical $x 5^{\circ}$ horizontal giving an instantaneous field of view (IFOV) of $0.76 \mathrm{mrad}$. The IR camera is located at about $1 \mathrm{~m}$ from the model yielding a typical spatial resolution on the skin of less than $1 \mathrm{~mm}$. 
http://dx.doi.org/10.21611/qirt.1994.022

The external surface of the model is painted uniformly in black. The thermograms are calibrated by measuring the internal surface temperature with T-type thermocouples flushmounted on the heated element and connected to the PC-controlled data acquisition system. To overcome the effect of the emissivity change due to the surface curvature the procedure followed consists in scanning the skin at different view angles by rotating the model around its axis. Preliminary tests have proved that three sub-thermograms, one from the top, one from the center and one from the bottom of the airfoil mock-up, are sufficient to reconstitute correctly the complete mapping of the thermal field. A typical example of such sub-thermograms is given in figure 4 . From these three pictures, a specific application DIP software performs image assembling and restoration, noise reduction by 2D-FFT and MTF - SRF corrections, if necessary, to reproduce the complete temperature field over the developed surface of the model. At steady state conditions, the local convective heat transfer coefficient $h_{c}$ is determined from the recovery temperature of the air jet $T \mathrm{jr}$, the internal surface temperature $T_{\mathrm{w}}$, and the Joule heating $q \mathrm{~J}$, according to the Newton relation:

$$
h_{c}(x, y)=\frac{q_{J}-q_{l}(x, y)}{T_{w}(x, y)-T_{j}}
$$

$q_{\ell}$ is the heat loss due to conduction in the skin, the natural convection and radiation between the external surface and the environment but also the radiation between the internal surface and the piccolo tube. Making use of the two-dimensional fin theory leads to the following expression:

$$
q_{\ell}=-e k \nabla^{2} T_{w}+h_{\theta}\left(T_{w}-T_{\theta}\right)+h_{1}\left(T_{w}-T_{p}\right)
$$

where $e$ and $k$ are the equivalent thickness and thermal conductivity of the skin, respectively; $h_{e}$ and $h_{i}$ are the global external and internal heat transfer coefficients modeling natural convection and thermal radiation effects.

The relations (1) and (2) show that $h_{\mathrm{c}}$ can e determined readily once the skin properties and temperature field $T_{w}$ are known. It is worthwhile noting that an evaluation of the conduction term shows that the data must be smoothed by using a twodimensional fast Fourier transform in order to eliminate the presence of spurious high frequency oscillations in the temperature field. Table I lists the typical contribution of the different heat transfer modes involved in the experiments. From a detailed error analysis it is found that the local uncertainty on

\begin{tabular}{|l|c|}
\hline Heat transfer mode & Percentage of qu \\
\hline $\begin{array}{l}\text { External natural } \\
\text { convection }\end{array}$ & 4 \\
\hline External radiation & 8 \\
\hline Skin conduction & 1 \\
\hline Internal radiation & 3 \\
\hline Jet convection & 84 \\
\hline
\end{tabular}

Table 1-Contribution of the heat transfer modes the $h_{c}$-value can reach $10 \%$. The practical lessons drawn from the above equations are twofold: first, even though the wall material is of low thermal conductivity and small thickness, it is mandatory to use a thermal sensor with high spatial resolution to resolve properly the conductive term. In this respect, infrared thermography is an appealing technique. Second, the heated surface should be uniformly coated with a film of large emissivity to avoid artificial distortion in the temperature field and difficult reflectivity corrections [5].

\section{Typical results}

Seven piccolo tubes are tested allowing to vary the normalized piccolo stand-off $Z / d$ from 9 to 16, the reduced spanwise $S_{\mathrm{p}} / d$ and chordwise spacing $S_{\mathrm{c}} / d$ between 15 
http://dx.doi.org/10.21611/qirt.1994.022

and 40 and 30 and 60 respectively. The Reynolds number, based on exit velocity $U_{1}$ and diameter $d$ of the jet, is varied from 4000 to 14000 .

A typical mapping of the convective heat transfer coefficient for an arrangement of four jet rows is shown in figure 5 . The abscissa and ordinate are normalized by the model length $I$ and the chordwise length $L$, respectively. The confinement effect of the two central rows is depicted by the highest $h_{c}$-values obtained in this zone. A sharp decrease of thermal exchange is also observed on the far intrados and extrados where the multijet effect is weak. The corresponding normalized $h c$-profiles along the chordwise directions $\mathrm{K} 1, \mathrm{~K} 10$ and $\mathrm{K} 20$ defined in figure 5, are plotted in figure 6 . Variations of the heat transfer coefficient as high as $80 \%$ can be expected in the impact area of the multijets. The slight increase of $h_{c}$ detected at the extremities is interpreted as the acceleration of the boundary layer as the flow approaches the slit.

The effect of the jet Reynoids number is illustrated by the $h_{c}$-profiles shown in figure 7 . The increase of $R e$ improves significantly the convective thermal exchange. At the same time it modifies the complex jet-to-jet interaction that takes place in the confined chamber formed by the skin and the baffles. Such a behavior is reflected by the change of the impingement positions observed as the Reynolds number varies. For engineering purposes, it is often more convenient to consider a mean heat transfer coefficient $\langle h\rangle$. To this end, a correlation is derived from the IR data in terms of a average Nusselt number $\langle N u\rangle=\langle h\rangle d / k_{a}$ calculated on the cell of the jet array. The results are shown in figure 8 where the dimensionless group $\left\langle N u>/ R e^{0.8} P r^{0.4}\right.$ is plotted versus the geometrical factor Go defined as following:

$$
G o=n_{j}^{a}\left[\frac{S_{c}}{d}\right]^{-\theta}\left[\frac{S_{p}}{d}\right]^{-b}\left[\frac{Z}{d}\right]^{-c}
$$

$n_{\mathrm{j}}$ is the number of jets in the cell. The best curve fit for these data leads to the following

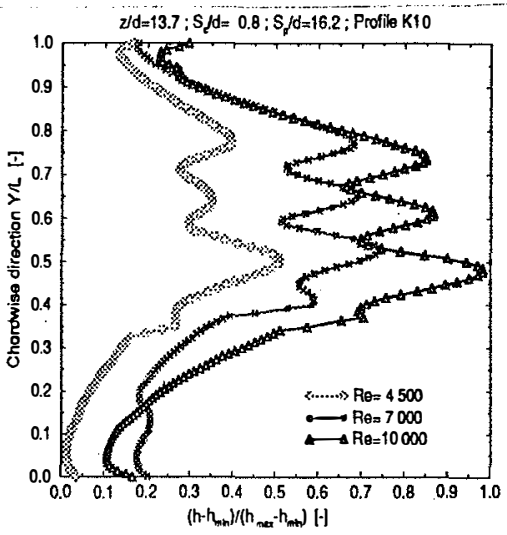

Fig. 7 - Effect of the Reynolds number

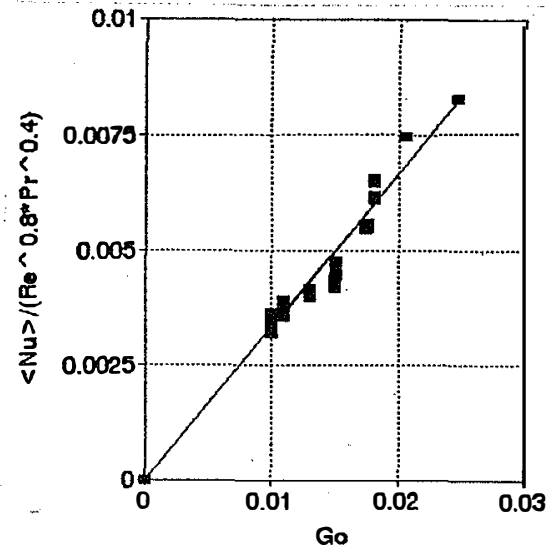

Fig. 8 - Average Nusselt

exponent-values $a=0.4, b=0.9$ and $c=0.1$. In the range investigated, the normalized stand-off distance has a small influence on the convective thermal exchange. The dominant parameter is the reduced spanwise jet spacing: as $S_{p} / d$ decreases the dead zone between the jet impact shrinks and the average heat transfer rises. 


\section{Numerical simulations}

Numerical simulations of the aerothermal behavior of the multijet system are performed to compute the heat transfer in the real conditions and make a predesign of the anti-icing configuration. To assess the validity of the numerical approach, the $\mathbb{I R}$ experiments are simulated and the heat transfer coefficients given by both methods are compared. The computations are done using the code FLUENT. The equations of mass, momentum and energy are solved by application of a finite volume technique. The turbulence model used is a $k-\varepsilon$ model based on the renormalization-group theory. Tridimensional simulations are conducted to represent accurately the flow field inside the leading edge.

To limit the number of the computational cells $(60,000)$, the flow field is computed between two chordwise symmetry planes. The boundary conditions applied on the domain are chosen to be as close as possible to the mock-up configuration. The temperature of the jets and the piccolo is fixed at the temperature of the air measured with the thermocouple. The baffles are adiabatic. However, a problem arises when fixing the boundary conditions at the leading edge. To be consistent, the experimental value of the internal heat flux, taking into account this external convection, should be applied locally along all the leading edge. Unfortunately, this is presently not possible with FLUENT. Therefore, to circumvent such a difficulty, the condition of a constant skin temperature is adopted.

A typical simulation of the tridimensional velocity field in the cove is represented on figure 9. The multijet system is characterized by a complex flow pattern. A typical comparison between the correlation experimental and predicted heat transfer coefficient is presented in figure 10. Despite the complexity of the problem, it can be concluded that the numerical simulation is in satisfactory agreement with the experimental results: the shape of the heat transfer distribution curve is well computed, however, it is noticed that the simulation under-estimates the IR data. New computations with a refined grid, different turbulence parameters and new boundary conditions are in progress to get closer to the experimental values.

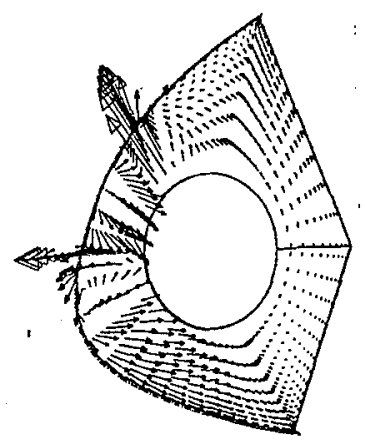

Plane view

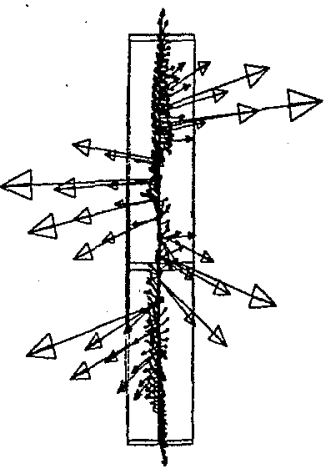

Front view

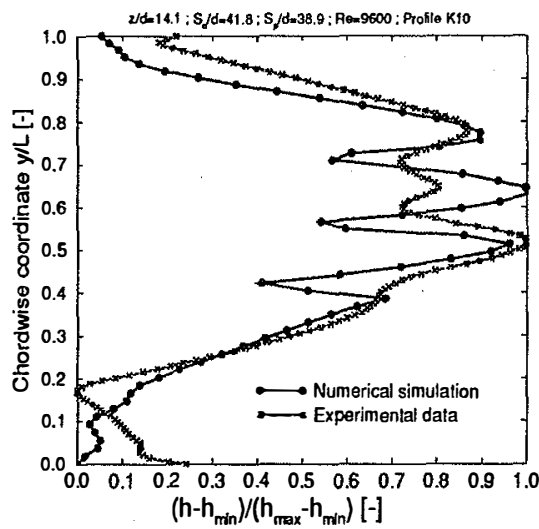

Fig. 9 - Velocity field in the multijet

Fig 10 - Comparison of numerical simulations and experimental results

\section{Conclusions}

Steady state heat transfer measurements in a thermal anti-icing system of an airplane wing are performed. The heated foil technique combined with two-dimensional fin theory 
http://dx.doi.org/10.21611/qirt.1994.022

and associated with quantitative infrared thermography and digital image processing constitutes a powerful experimental tool to determine the mapping of the convective heat transfer coefficient resulting from impingement of multijets on the internal surface of the airfoil. IR data emphasize the heat transfer enhancement induced by the increase of the jet Reynolds number and the reduction of the spanwise jet spacing. A general correlation of the average cell Nusselt number including the effect of the flow parameters and the geometrical factor is proposed. Tridimensional numerical simulations are performed with the FLUENT code. Despite the complexity of the flow phenomena and the stiff character of boundary conditions involved, satisfactory agreement is found between the predictions and the experimental results. However, further refinements of the numerical simulations already in progress are mandated.

\section{REFERENCES}

[1] MARTIN (H). - Heat and Mass Transfer between impinging gas jets and solid surfaces. Adv. Heat Transfer, Vol. 13, 1977, p. 1-60.

[2] Aircraft lcing Handbook. DOT/FAA/CT-88/8-2, FAA Technical Center, NJ08405, MARCA 1991.

[3] BUCHLIN (J-M). - Digital image processing technique applied to fluid dynamic problems. AGARD PEP 67th Symp. on Advanced Instrumentation for Aero Engine Components, Philadelphia, USA, May 1986.

[4] BUCHLIN (J-M) and DUBOIS (M). - Heat transfer of impinging multijet system. An application of the quantitative themography. Quantitative Infrared Thermography QIRT 92, EUROTHERM Seminar 27, Châtenay-Malabry, France, July 7-9, 1992, p. 117-120.

[5] BUCHLIN (J-M) and ARTS (T). - Mesure de températures de surface par cristaux liquides et thermographie infrarouge. VKI-Preprint 1991-31, 'Journées d'Etudes et d'Exposition; Développements récents en transfert de chaleur. Applications et produits nouveaux.' Société Belge des Mécaniciens, Bruxelles, 16-17 Octobre 1991. 